\title{
Bicomplexes, Integrable Models, and Noncommutative Geometry
}

\author{
Aristophanes Dimakis \\ Department of Mathematics, University of the Aegean \\ GR-83200 Karlovasi, Samos, Greece \\ Folkert Müller-Hoissen \\ Max-Planck-Institut für Strömungsforschung \\ Bunsenstrasse 10, D-37073 Göttingen, Germany
}

\begin{abstract}
We discuss a relation between bicomplexes and integrable models, and consider corresponding noncommutative (Moyal) deformations. As an example, a noncommutative version of a Toda field theory is presented.
\end{abstract}

\section{Introduction}

Soliton equations and integrable models are known to possess a vanishing curvature formulation depending on a parameter, say $\lambda$ (cf [1], for example). This geometric formulation of integrable models is easily extended [2, 3] to generalized geometries, in particular in the sense of noncommutative geometry where, on a basic level, the algebra of differential forms (over the algebra of smooth functions) on a manifold is generalized to a differential calculus over an associative (and not necessarily commutative) algebra $\mathcal{A}$.

A bicomplex associated with an integrable model is a special case of a zero curvature formulation. More precisely, let $\mathcal{M}=\bigoplus_{r \geq 0} \mathcal{M}^{r}$ be an $\mathbb{N}_{0}$-graded linear space (over $\mathbb{R}$ or $\mathbb{C}$ ) and d, $\delta: \mathcal{M}^{r} \rightarrow \mathcal{M}^{r+1}$ two linear maps satisfying

$$
\mathrm{d}^{2}=0, \quad \delta^{2}=0, \quad \mathrm{~d} \delta+\delta \mathrm{d}=0
$$

(typically as a consequence of certain field equations). Then $(\mathcal{M}, \mathrm{d}, \delta)$ is called a bicomplex. Special examples are bi-differential calculi [3]. However, we do not need $\mathrm{d}$ and $\delta$ to be graded derivations (into some bimodule), i.e., they do not have to satisfy the Leibniz rule.

Given a bicomplex, there is an iterative construction of "generalized conserved densities" in the sense of $\delta$-closed elements of the bicomplex (see section 2). In some examples they reproduce directly the conserved quantities of an integrable model. In other examples, the

\footnotetext{
${ }^{1}$ In terms of $\mathrm{d}_{\lambda}=\delta-\lambda \mathrm{d}$ with a constant $\lambda$, the three bicomplex equations are combined into the single zero curvature condition $\mathrm{d}_{\lambda}^{2}=0$ (for all $\lambda$ ).
} 
relation is less direct. Anyway, the existence of such a chain of $\delta$-closed elements is clearly a distinguished feature of the model with which the bicomplex is associated.

Noncommutative examples are in particular obtained by starting with a classical integrable model, deforming an associated bicomplex by replacing the ordinary product of functions with the Moyal *-product and thus arriving at a noncommutative model. As an example, a noncommutative extension of a Toda field theory is considered in section 3. Field theory on noncommutative spaces has gained more and more interest during the last years. A major impulse came from the discovery that a noncommutative gauge field theory arises in a certain limit of string, D-brane and $\mathrm{M}$ theory (see [5] and the references cited there). We also refer to [6] for some work on Moyal deformations of integrable models.

\section{The bicomplex linear equation}

Let us assume that, for some $s \in \mathbb{N}$, there is a (nonvanishing) $\chi^{(0)} \in \mathcal{M}^{s-1}$ with $\mathrm{d} J^{(0)}=0$ where $J^{(0)}=\delta \chi^{(0)}$. Defining $J^{(1)}=\mathrm{d} \chi^{(0)}$, we have $\delta J^{(1)}=-\mathrm{d} \delta \chi^{(0)}=0$ using (1.1). If the $\delta$-closed element $J^{(1)}$ is $\delta$-exact, this implies $J^{(1)}=\delta \chi^{(1)}$ with some $\chi^{(1)} \in \mathcal{M}^{s-1}$. Next we define $J^{(2)}=\mathrm{d} \chi^{(1)}$. Then $\delta J^{(2)}=-\mathrm{d} \delta \chi^{(1)}=-\mathrm{d} J^{(1)}=-\mathrm{d}^{2} \chi^{(0)}=0$. If the $\delta$-closed element $J^{(2)}$ is $\delta$-exact, then $J^{(2)}=\delta \chi^{(2)}$ with some $\chi^{(2)} \in \mathcal{M}^{s-1}$. This can be iterated further and leads to a possibly infinite chain (see the figure below) of elements $J^{(m)}$ of $\mathcal{M}^{s}$ and $\chi^{(m)} \in \mathcal{M}^{s-1}$ satisfying

$$
J^{(m+1)}=\mathrm{d} \chi^{(m)}=\delta \chi^{(m+1)} .
$$

More precisely, the above iteration continues from the $m$ th to the $(m+1)$ th level as long as $\delta J^{(m)}=0$ implies $J^{(m)}=\delta \chi^{(m)}$ with an element $\chi^{(m)} \in \mathcal{M}^{s-1}$. Of course, there is no obstruction to the iteration if $H_{\delta}^{s}(\mathcal{M})$ is trivial, i.e., when all $\delta$-closed elements of $\mathcal{M}^{s}$ are $\delta$-exact. But in general the latter condition is too strong, though in several examples it can indeed be easily verified [3]. Introducing

$$
\chi=\sum_{m \geq 0} \lambda^{m} \chi^{(m)}
$$

with a parameter $\lambda$, the essential ingredients of the above iteration procedure are summarized in the linear equation associated with the bicomplex:

$$
\delta\left(\chi-\chi^{(0)}\right)=\lambda \mathrm{d} \chi .
$$

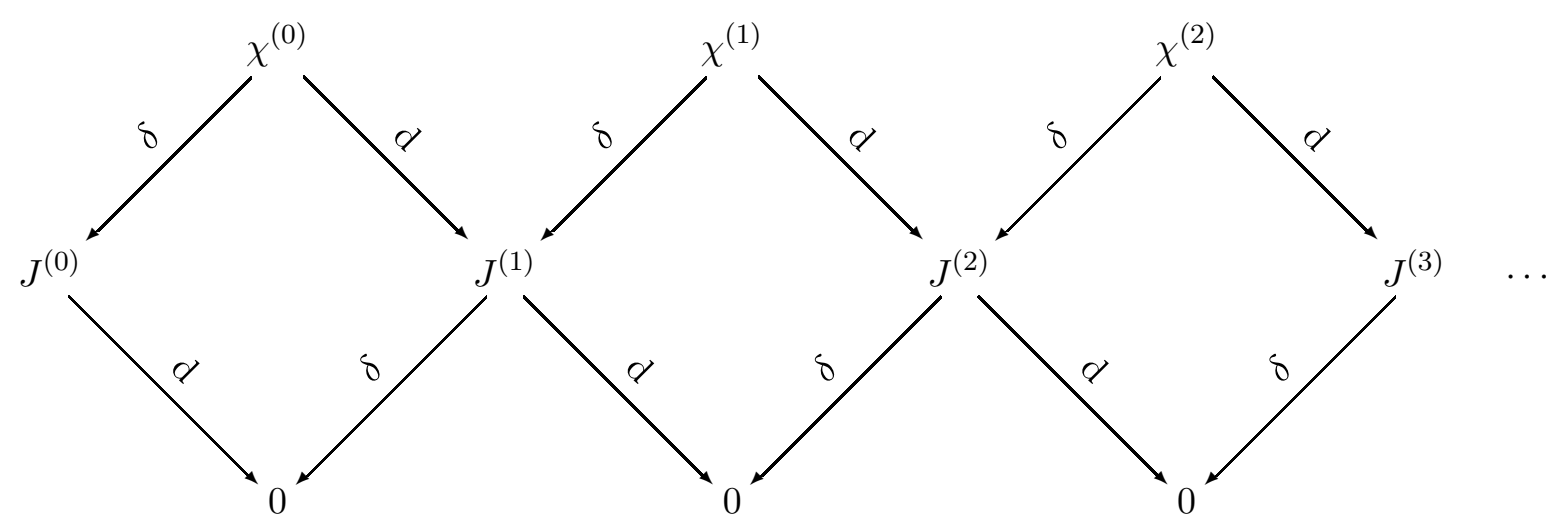


Given a bicomplex, we may start with the linear equation (2.3). Let us assume that it admits a (non-trivial) solution $\chi$ as a (formal) power series in $\lambda$. The linear equation then leads to $\delta \chi^{(m)}=\mathrm{d} \chi^{(m-1)}$. As a consequence, the $J^{(m+1)}=\mathrm{d} \chi^{(m)}$ are $\delta$-exact. Therefore, even if the cohomology $H_{\delta}^{s}(\mathcal{M})$ is not trivial, the solvability of the linear equation ensures that the $\delta$-closed $J^{(m)}$ appearing in the iteration are $\delta$-exact.

In all the examples which we presented in [3], 1], the bicomplex space can be chosen as $\mathcal{M}=\mathcal{M}^{0} \otimes \Lambda$ where $\Lambda=\bigoplus_{r=0}^{n} \Lambda^{r}$ is the exterior algebra of an $n$-dimensional vector space with a basis $\xi^{r}, r=1, \ldots, n$, of $\Lambda^{1}$. It is then sufficient to define the bicomplex maps $\mathrm{d}$ and $\delta$ on $\mathcal{M}^{0}$ since via

$$
\mathrm{d}\left(\sum_{i_{1}, \ldots, i_{r}=1}^{n} \phi_{i_{1} \ldots i_{r}} \xi^{i_{1}} \cdots \xi^{i_{r}}\right)=\sum_{i_{1}, \ldots, i_{r}=1}^{n}\left(\mathrm{~d} \phi_{i_{1} \ldots i_{r}}\right) \xi^{i_{1}} \cdots \xi^{i_{r}}
$$

(and correspondingly for $\delta$ ) they extend as linear maps to the whole of $\mathcal{M}$.

\section{Noncommutative deformation of a Toda model}

The $*$-product on the space $\mathcal{F}$ of smooth functions of two coordinates $x$ and $t$ is given by

$$
f * h=m \circ e^{\theta P / 2}(f \otimes h)=f h+\frac{\theta}{2}\{f, h\}+\mathcal{O}\left(\theta^{2}\right)
$$

where $\theta$ is a parameter, $m(f \otimes h)=f h$ and $P=\partial_{t} \otimes \partial_{x}-\partial_{x} \otimes \partial_{t}$. Furthermore, $\{$,$\} is the$ Poisson bracket, i.e., $\{f, h\}=\left(\partial_{t} f\right) \partial_{x} h-\left(\partial_{x} f\right) \partial_{t} h$. For the calculations below it is helpful to notice that partial derivatives are derivations of the algebra $(\mathcal{F}, *)$.

A bicomplex associated with an integrable model can be deformed by replacing the ordinary product of functions with the noncommutative $*$-product. This then induces a deformation of the integrable model with very special properties since the iterative construction of generalized conservation laws still works. As a specific example, we construct a noncommutative extension of the Toda field theory on an open finite one-dimensional lattice. Other examples can be obtained in the same way.

Let us start from the trivial bicomplex which is determined by

$$
\delta \phi=\left(\partial_{t}-\partial_{x}\right) \phi \xi^{1}+(S-I) \phi \xi^{2}, \quad \mathrm{~d} \phi=-S^{T} \phi \xi^{1}+\left(\partial_{t}+\partial_{x}\right) \phi \xi^{2}
$$

where $\phi$ is a vector with $n$ components (which are functions) and $S^{T}$ the transpose of

$$
S=\sum_{i=1}^{n-1} E_{i, i+1}, \quad\left(E_{i, j}\right)^{k}{ }_{l}=\delta_{i}^{k} \delta_{j, l}
$$

Let $G$ be an $n \times n$ matrix of functions which is invertible in the sense $G^{-1} * G=I$ where $I$ is the $n \times n$ unit matrix. Now we introduce a "dressing" for $\mathrm{d}$ :

$$
\mathrm{D} \phi=G^{-1} * \mathrm{~d}(G * \phi)=-(L * \phi) \xi^{1}+\left(\partial_{t}+\partial_{x}+M *\right) \phi \xi^{2}
$$

where

$$
L=G^{-1} * S^{T} * G, \quad M=G^{-1} *\left(G_{t}+G_{x}\right) .
$$


Note that $\mathrm{D}^{2} \phi=G^{-1} * \mathrm{~d}^{2}(G * \phi)=0$. The only nontrivial bicomplex equation is $\delta \mathrm{D}+\mathrm{D} \delta=0$ which reads

$$
M_{t}-M_{x}=L * S-S * L .
$$

Hence, if this equation holds, then $\left(\mathcal{F}^{n} \otimes \Lambda, \mathrm{D}, \delta\right)$ is a bicomplex. Let us now choose

$$
G=\sum_{i=1}^{n} G_{i} E_{i i}
$$

with functions $G_{i}$ for which the invertibility assumption requires $G_{i}^{-1} * G_{i}=1$. Then

$$
L=\sum_{i=1}^{n-1} G_{i+1}^{-1} * G_{i} E_{i+1, i}, \quad M=\sum_{i=1}^{n} M_{i} E_{i i}, \quad M_{i}=G_{i}^{-1} *\left(\partial_{t}+\partial_{x}\right) G_{i}
$$

Writing

$$
G_{i}=e^{q_{i}}\left(1+\theta \tilde{q}_{i}\right)+\mathcal{O}\left(\theta^{2}\right)
$$

we have $G_{i}^{-1}=e^{-q_{i}}\left(1-\theta \tilde{q}_{i}\right)+\mathcal{O}\left(\theta^{2}\right)$ and it follows from (3.6) that the functions $q_{i}$ have to solve the Toda field theory equations

$$
\begin{aligned}
\left(\partial_{t}^{2}-\partial_{x}^{2}\right) q_{i} & =e^{q_{i-1}-q_{i}}-e^{q_{i}-q_{i+1}} \quad i=2, \ldots, n-1 \\
\left(\partial_{t}^{2}-\partial_{x}^{2}\right) q_{1} & =-e^{q_{1}-q_{2}}, \quad\left(\partial_{t}^{2}-\partial_{x}^{2}\right) q_{n}=e^{q_{n-1}-q_{n}} .
\end{aligned}
$$

Furthermore, the functions $\tilde{q}_{i}$ are subject to the following linear equations,

$$
\begin{aligned}
\left(\partial_{t}^{2}-\partial_{x}^{2}\right) \tilde{q}_{1} & =\left\{\partial_{t} q_{1}, \partial_{x} q_{1}\right\}-e^{q_{1}-q_{2}}\left(\tilde{q}_{1}-\tilde{q}_{2}\right) \\
\left(\partial_{t}^{2}-\partial_{x}^{2}\right) \tilde{q}_{i} & =\left\{\partial_{t} q_{i}, \partial_{x} q_{i}\right\}+e^{q_{i-1}-q_{i}}\left(\tilde{q}_{i-1}-\tilde{q}_{i}\right)-e^{q_{i}-q_{i+1}}\left(\tilde{q}_{i}-\tilde{q}_{i+1}\right) \\
\left(\partial_{t}^{2}-\partial_{x}^{2}\right) \tilde{q}_{n} & =\left\{\partial_{t} q_{n}, \partial_{x} q_{n}\right\}+e^{q_{n-1}-q_{n}}\left(\tilde{q}_{n-1}-\tilde{q}_{n}\right) .
\end{aligned}
$$

A 1-form $J=P \xi^{1}+R \xi^{2}$ is $\delta$-closed iff $\left(\partial_{t}-\partial_{x}\right) R=(S-I) P$. For $J=\lambda \mathrm{D} \chi(\operatorname{cf}(2.1))$ we have $P=-\lambda L * \chi$ and $R=\lambda\left(\partial_{t}+\partial_{x}+M *\right) \chi$ and thus

$$
\partial_{t}\left[\lambda\left(\partial_{t}+M_{i} *\right) \chi_{i}\right]=\partial_{x}\left[\lambda\left(\partial_{x}+M_{i} *\right) \chi_{i}\right]+P_{i+1}-P_{i}
$$

$(i=1, \ldots, n)$ where we have to set $P_{n+1}=0$. Using $P_{1}=0$, we find that

$$
Q=\lambda \int d x \sum_{i=1}^{n}\left(\partial_{t} \chi_{i}+G_{i}^{-1} *\left[\left(\partial_{t}+\partial_{x}\right) G_{i}\right] * \chi_{i}\right)
$$

is conserved, i.e., $d Q / d t=0$, provided that the expressions $\partial_{x} \chi_{i}+M_{i} * \chi_{i}$ vanish at $x= \pm \infty$. In order to further evaluate this expression, we have to explore the linear system associated with the bicomplex. Choosing $\chi^{(0)}=\sum_{i=1}^{n} e_{i}$, where $e_{i}$ is the vector with components $\left(e_{i}\right)_{j}=\delta_{i j}$, we have $\delta \chi^{(0)}=-e_{n} \xi^{2}$ and $\mathrm{D} \delta \chi^{(0)}=0$. Now we find

$$
\begin{aligned}
Q^{(1)} & =\int d x \sum_{i=1}^{n} M_{i}=\int d x \sum_{i=1}^{n} G_{i}^{-1} *\left(\partial_{t}+\partial_{x}\right) G_{i} \\
& =\int d x \sum_{i=1}^{n} \partial_{t} q_{i}+\theta \int d x \sum_{i=1}^{n}\left(\left(\partial_{t}+\partial_{x}\right) \tilde{q}_{i}+\frac{1}{2}\left\{\left(\partial_{t}+\partial_{x}\right) q_{i}, q_{i}\right\}\right)+\mathcal{O}\left(\theta^{2}\right)
\end{aligned}
$$


where we assumed that the $q_{i}$ vanish at $x= \pm \infty$. The linear system $\delta\left(\chi-\chi^{(0)}\right)=\lambda \mathrm{D} \chi$ reads

$$
\left(\partial_{t}-\partial_{x}\right) \chi_{1}=0, \quad\left(\partial_{t}-\partial_{x}\right) \chi_{i}=-\lambda G_{i}^{-1} * G_{i-1} * \chi_{i-1} \quad i=2, \ldots, n
$$

and

$$
\begin{aligned}
\chi_{i+1}-\chi_{i} & =\lambda\left(\partial_{t}+\partial_{x}+M_{i} *\right) \chi_{i} \quad i=1, \ldots, n-1 \\
\chi_{n} & =1-\lambda\left(\partial_{t}+\partial_{x}+M_{n} *\right) \chi_{n} .
\end{aligned}
$$

Using $\chi_{i}^{(0)}=1$, (3.16) yields $\chi_{i+1}^{(1)}-\chi_{i}^{(1)}=M_{i}$ and thus巳 $\chi_{i}^{(1)}=-\sum_{k=i}^{n} M_{k}$. After some manipulations and using (3.10), we obtain

$$
\begin{aligned}
Q^{(2)}= & -\int d x \sum_{i=1}^{n} \sum_{k=i}^{n}\left(\partial_{t} M_{k}+M_{i} * M_{k}\right) \\
= & -\int d x\left(\sum_{i=1}^{n-1} e^{q_{i}-q_{i+1}}+\frac{1}{2}\left(\sum_{i=1}^{n}\left(\partial_{t}+\partial_{x}\right) q_{i}\right)^{2}-\frac{1}{2} \sum_{i=1}^{n}\left[\left(\partial_{t}+\partial_{x}\right) q_{i}\right]^{2}\right) \\
& +\mathcal{O}(\theta)
\end{aligned}
$$

where to first order in $\theta$ already a rather complicated expression emerges. At 0 th order in $\theta$ one recovers the known conserved charges of the Toda theory.

Infinite-dimensional integrable models possess an infinite set of conserved currents. In contrast to previous approaches to deformations of integrable models (see [6], for example), our approach guarantees, via deformation of the bicomplex associated with an integrable model, that this infinite tower of conservation laws survives the deformation.?

\section{References}

[1] Faddeev L D and Takhtajan L A, Hamiltonian Methods in the Theory of Solitons (Berlin: Springer, 1987).

[2] Dimakis A and Müller-Hoissen F, Soliton equations and the zero curvature condition in noncommutative geometry J. Phys. A: Math. Gen. 29 (1996) 7279-7286; Integrable discretizations of chiral models via deformation of the differential calculus $J$. Phys. A: Math. Gen. 29 (1996) 5007-5018; Noncommutative geometry and integrable models Lett. Math. Phys. 39 (1997) 69-79; Noncommutative geometry and a class of completely integrable models Czech. J. Phys. 48 (1998) 1319-1324.

\footnotetext{
${ }^{2} \mathrm{~A}$ "constant of integration" can be added on the rhs. But this would simply lead to an additional term proportional to $Q^{(1)}$ in (3.18).

${ }^{3}$ Deforming a Hamiltonian system which is (Liouville) integrable so that the conserved charges are in involution with respect to a symplectic structure, the question arises whether there is a corresponding deformation of the symplectic and Hamiltonian structure such that the deformation preserves the involution property.
} 
[3] Dimakis A and Müller-Hoissen F, Bi-differential calculi and integrable models J. Phys. A 33 (2000) 957-974.

[4] Dimakis A and Müller-Hoissen F, Bi-differential calculus and the KdV equation, mathph/9908016, to appear in Rep. Math. Phys.; Bicomplexes and finite Toda lattices, solvint/9911006, to appear in Quantum Theory and Symmetries, eds. H.-D. Doebner, V.K. Dobrev, J.-D. Hennig and W. Lücke (Singapore: World Scientific); Bicomplexes and integrable models, in preparation.

[5] Seiberg N and Witten E, String theory and noncommutative geometry, hep-th/9908142, JHEP 09 (2000) 032.

[6] Strachan I A B, A geometry for multidimensional integrable systems J. Geom. Phys. 21 (1997) 255-278; García-Compeán H, Plebański J F and Przanowski M, Geometry associated with self-dual Yang-Mills and the chiral model approaches to self-dual gravity Acta Phys. Polon. B 29 (1998) 549-571; Takasaki K, Anti-self-dual Yang-Mills equations on noncommutative spacetime, hep-th/0005194. 